\title{
Torsion tests on small and large-scale RHS with circular wall cut-outs
}

\author{
D.J.Ridley-Ellis, J.S.Owen \& G.Davies \\ University of Nottingham, Nottingham, United Kingdom
}

\begin{abstract}
The results of small and large-scale torsion tests on rectangular section tubes with circular wall perforations are presented and discussed. Perforations are shown to cause the torsional resistance to be reduced by up to $60 \%$ and stiffness to be reduced by up to $40 \%$. The perforated zone is shown to be much more flexible than the unperforated section, which results in severe deformations in this region. Small holes cause small reductions in the torsional resistance and stiffness, but in some instances result in a large reduction in the torsional ductility of the member. The experimental results are favourably compared to the predictions of finite element models, which are then used to demonstrate the importance of the boundary conditions for the asymmetrical cases, and to study in more detail the stress distributions around the openings.
\end{abstract}

\section{INTRODUCTION}

The inherent torsional rigidity of Rectangular Hollow Sections (RHS) means that they are particularly suitable for use as edge beams in Slim Floor type construction. Slim Floor systems such as Slimdek (Mullett 1997) allow basic building services to be integrated within the structural depth. At the perimeter of the building, these services may be required to pass within the void of the edge beam, or through the edge beam to the building exterior. In both cases, openings are required in the webs of the edge beam, which affect its structural performance.

Rules for the design of I-beams with web openings in flexure are readily available, but there are currently no rules for the design of perforated RHS. Therefore, a thorough investigation into the behaviour of RHS with circular wall openings in torsion, bending and shear has been carried out at the University of Nottingham (Ridley-Ellis 2000), funded by Corus Tubes and the University of Nottingham. A combination of laboratory testing and finite element analysis (FEA) was used to develop an understanding of the fundamental structural behaviour of perforated RHS subject to combined actions, and to develop the basis for a set of design rules.

This paper summarises the results of small-scale and full-scale torsion tests performed on RHS that have circular openings placed at mid-depth in the walls and compares the measurements to predictions made by FEA. It extends the work published previ- ously (Ridley-Ellis et al 1998), through the inclusion of the results of full-scale tests, extended discussion and analysis, and the use of improved FEA models. It also proposes methods of approximating the reduced linear torsional response as well as the magnitudes of stress concentrations that occur around an opening. Two cases of perforated section were considered, depending on whether just one wall, or two opposing walls contained holes. For the cases where two walls contained holes, those holes were placed symmetrically and were of equal diameter.

\section{DETAILS OF LABORATORY TESTS}

\subsection{Full-scale tests}

The full-scale torsion testing rig comprised of a pivoted rotating beam and a fixed reaction beam, between which a specimen was caused to twist about its longitudinal axis (Fig. 1). A matched pair of hydraulic jacks, mounted on guide columns, applied the torsional action through the rotating beam, which was supported on the axis of the rig by a bearing. The torque was transmitted to the two metre long specimen through bolted connections at both ends.

The torque was measured by using shear strain gauges on the outside surface of a hollow circular shaft between the specimen and the rotating beam (Calibrated up to $95 \mathrm{kNm}$ ). The rotation was measured at each end of the specimen using gravity inclinometers mounted on the connecting plates. 


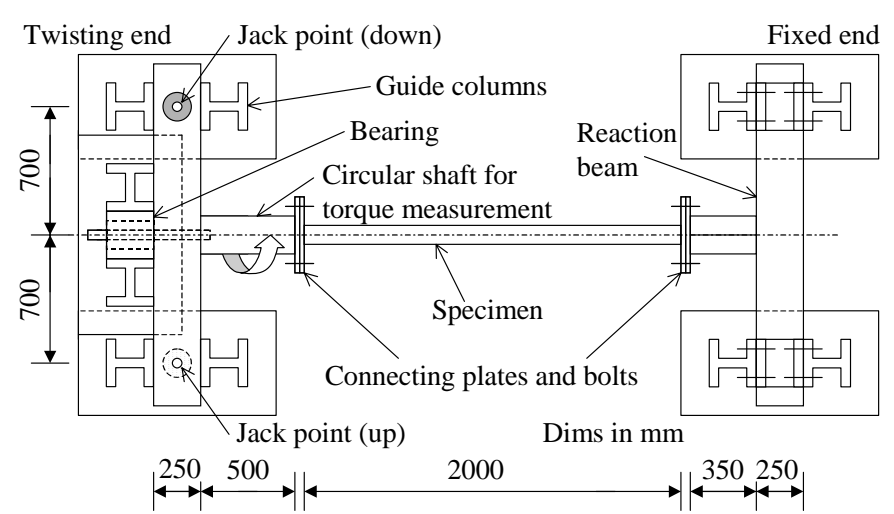

Figure 1. The full-scale torsion testing rig.

Table 1. Full-scale specimens tested.

\begin{tabular}{|c|c|c|c|c|}
\hline $\begin{array}{l}\mathrm{N}^{0} \text { of } \\
\text { tests }\end{array}$ & $\begin{array}{l}\text { Section } \\
\text { BSEN10210 }\end{array}$ & $\begin{array}{l}\mathrm{N}^{0} \text { of } \\
\text { holes }\end{array}$ & $\begin{array}{l}\text { Hole dia } \\
(\mathrm{mm})\end{array}$ & $\begin{array}{l}\text { Hole size } \\
\text { ratio }(\Phi)\end{array}$ \\
\hline 1 & 200x100x8 S275 & 0 & - & 0 \\
\hline 1 & " & 1 & 165.0 & 0.94 \\
\hline 1 & " & 2 & 165.0 & 0.94 \\
\hline 2 & 200x100x8 S355 & 0 & - & 0 \\
\hline 1 & " & 1 & 165.0 & 0.94 \\
\hline 1 & $"$ & 2 & 165.0 & 0.94 \\
\hline 1 & $\begin{array}{l}150 \times 150 \times 6.3 \\
S 275\end{array}$ & 0 & - & 0 \\
\hline 1 & " & 1 & 99.0 & 0.76 \\
\hline 1 & " & 2 & 99.0 & 0.76 \\
\hline 1 & $\begin{array}{l}150 \times 150 \times 6.3 \\
\text { S355 }\end{array}$ & 0 & - & 0 \\
\hline 1 & $"$ & 1 & 99.0 & 0.76 \\
\hline 1 & " & 2 & 99.0 & 0.76 \\
\hline 1 & " & 2 & 38.1 & 0.29 \\
\hline
\end{tabular}

The full-scale specimens tested are listed in Table 1. Four different hot-finished bars were used in the manufacture of the specimens (two section sizes each of two different grades). The grade S275J2H material was manufactured by British Steel (now Corus) and the grade S355J2H material was manufactured by Vallourec and Mannesmann Tubes. Further details of the testing apparatus and procedure are given elsewhere (Ridley-Ellis et al 2002 \& 2003).

\subsection{Small-scale tests}

The small-scale torsion tests were conducted on lengths of square section cold-formed steel tube, the dimensions of which are shown in Figure 2. The cross-section of the tube (slenderness 23.6) was close to being geometrically similar to the RHS $150 \times 150 \times 6.3$ used in the full-scale torsion study (slenderness 20.8). The specimens were $300 \mathrm{~mm}$ in length, but were restrained at the ends by the stiff clamping plates and solid inserts, which provided connectivity with an Avery 6609 CGG torsiontesting machine $(1.7 \mathrm{kNm})$. In all the tests, the length of the portion of the specimen that was free to twist under load was $240 \mathrm{~mm}$. The torque was applied at a constant rate of twist $\left(3^{1} / 3\right.$ degrees per minute).

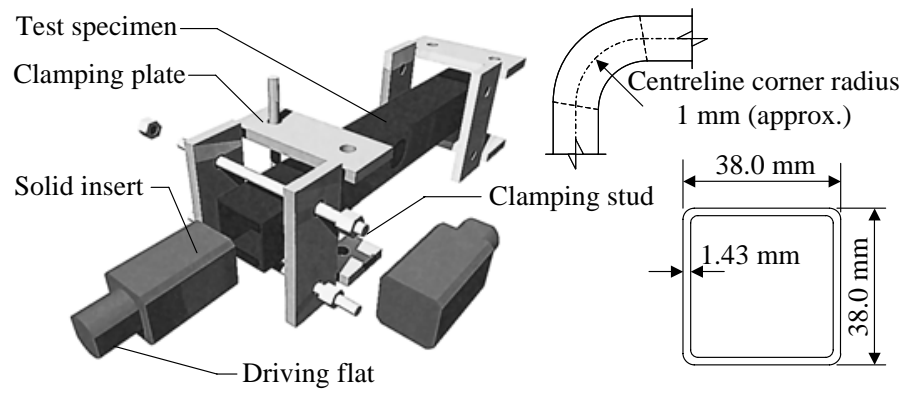

Figure 2. Small-scale torsion testing specimen.

Table 2. Small-scale specimens tested.

\begin{tabular}{llrrr}
\hline $\begin{array}{l}\mathrm{N}^{0} \text { of } \\
\text { tests }\end{array}$ & $\begin{array}{l}\text { Section } \\
\text { (measured dims) }\end{array}$ & $\begin{array}{l}\mathrm{N}^{0} \text { of } \\
\text { holes }\end{array}$ & $\begin{array}{l}\text { Hole dia } \\
(\mathrm{mm})\end{array}$ & $\begin{array}{l}\text { Hole size } \\
\text { ratio }(\Phi)\end{array}$ \\
\hline 6 & $38.0 \times 38.0 \times 1.43$ & 0 & - & 0 \\
2 & $"$ & 1 & 5 & 0.15 \\
2 & $"$ & 1 & 10 & 0.30 \\
2 & $"$ & 1 & 15 & 0.44 \\
2 & $"$ & 1 & 20 & 0.59 \\
2 & $"$ & 1 & 25 & 0.74 \\
2 & $"$ & 1 & 30 & 0.89 \\
2 & $"$ & 2 & 5 & 0.15 \\
2 & $"$ & 2 & 10 & 0.30 \\
2 & $"$ & 2 & 15 & 0.44 \\
2 & $"$ & 2 & 20 & 0.59 \\
2 & $"$ & 2 & 25 & 0.74 \\
2 & $"$ & 2 & 30 & 0.89 \\
\hline
\end{tabular}

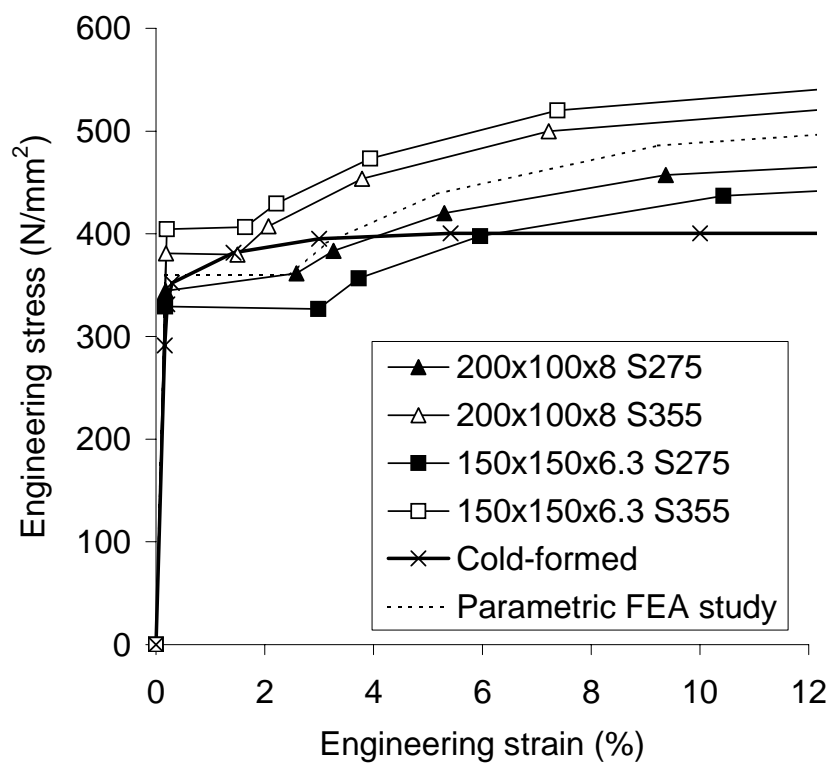

Figure 3. Material model properties for FEA.

The small-scale specimens tested are listed in Table 2. Three different cold-formed bars were used in the manufacture of the specimens, which were found to have nearly identical material properties and cross-section dimensions. Further details of the testing apparatus and procedure are given in reference Ridley-Ellis et al 1998. 


\section{DETAILS OF FINITE ELEMENT ANALYSIS}

\subsection{Modelling of the full-scale tests}

In order to provide additional information about the effect of the wall openings, the full-scale laboratory tests were replicated using the ABAQUS v5.8.1 FEA program (HKS 2000). Four noded shell elements (S4R) were chosen to create the models because they were able to model the changes in thickness that occurred due to high strains around the opening. A sensitivity study indicated that between 6200 and 8300 of these elements were required, depending on the experimental configuration. The models were based on the actual measured dimensions of the test specimens and included the corner radii of the tube.

The connecting plates were included in the models and the support conditions were defined by constraint equations describing the permitted displacements in the nodal degrees of freedom. The twisting action was applied via an angular displacement to one end of the model RHS.

Both material and geometric non-linearities were accounted for in the models. The non-linear material models used (Fig. 3) were based on the results of a comprehensive series of direct tensile tests, which showed that the steel in the tubes contained a long yield plateau typical of hot finished steel. Typically, strain hardening did not begin until engineering strain was in the order of $2.5 \%$.

\subsection{Modelling of the small-scale tests}

The small-scale tests were also replicated using FEA. The models used the same element type as the full-scale tests, but required fewer (3100 to 5900) due to the small-scale specimens being much shorter in relation to their cross-section. The solid inserts and clamping plates were not modelled directly, but were represented by constraint equations specifying the permitted displacements of the nodes in the cross-section at the ends of the model tube.

The non-linear material used in the FEA models (Fig. 3) was based on the results of a series of direct tensile tests. However, the Young's modulus of the material of the tubes used in the small-scale tests proved to be difficult to measure accurately using tensile tests, and the value used in the model was some $10 \%$ lower than the standard value for steel, and $15 \%$ lower than the value calculated from the results of flexural tests on a $5.5 \mathrm{~m}$ length of the complete tube.

The yield strength of the material of the coldformed tube was similar to those of the two grade S275 hot-finished tubes, although the tensile strength was $14 \%$ lower. Unlike the steel of the hotfinished tubes, the steel of the cold-formed tube had no yield plateau.

\subsection{Modelling for the parametric study}

In order to provide further information about stress concentrations, and the importance of relative hole diameter and tube aspect ratio, a parametric study was undertaken using FEA models modified from those used to replicate the laboratory tests. Sensitivity studies showed that the models required between 3600 and 4300 four noded shell elements.

Three section sizes were selected for the parametric study (250x250x10, 300x200x12 \& 200x100x8). These had similar wall (most slender is perforated) slendernesses (22) to the sections used in the laboratory tests, and had aspect ratios (1.0, 1.5 and 2.0) representing the European Standard range (BS EN 10210-2:1997).

The parametric models were used in the wider project to investigate the behaviour of edge beams under combinations of loads, and the boundary conditions were selected to recreate the conditions typical in service. Fixed and active boundary conditions were achieved by linking, via constraint equations, the displacements of the cross-sections of each end of the RHS to the displacements of a single node. The models used to study the action of torsion alone (those results presented here) were displaced at each end of the specimen by rotations about the longitudinal axis. The rotations were equal in magnitude, but opposite in direction, so as to produce a uniform torsional moment over the full length of the model RHS. No external bending moments or shear forces were applied, although flexure of the asymmetric sections was resisted by fixed boundary conditions at the ends of the tube. The model RHS were of sufficient length $(2.0 \mathrm{~m})$ to ensure that end effects did not influence the behaviour of the perforated zone for small plastic rotations.

A standardised material model was used in the parametric study (Fig. 3), which had typical hotfinished yield plateau and strain-hardening behaviour. The material fits the criteria for both the S275 and S355 material grades.

\section{DISCUSSION OF RESULTS}

\subsection{Measurements of torque-twist relationships}

The torque rotation curves for the laboratory torsion tests are shown in Figures 4,5 and 6. The torque and rotation have been normalised against the theoretical elastic torque $\left(T_{e l}\right)$ and rotation $\left(\theta_{e l}\right)$ of the corresponding unperforated section as calculated by simplified thick-wall theory (see Ridley-Ellis et al 2002 and 2003).

The results indicate that large openings in the walls cause a significant reduction in both the torsional stiffness and capacity. The reduction in stiffness is due to the perforated zone being much more flexible than the unperforated beam, and severe de- 
formations were observed around the openings (e.g. Fig. 7). The reduction in stiffness was lower for the specimens with square cross-sections than for the specimens with rectangular cross-sections, as, in the former, the perforated walls contributed a smaller proportion toward the total resistance of the crosssection to the applied torque.

Observation of the Lüders lines in the steel indicated that, for the perforated specimens, yielding occurred at low magnitudes of torque, due to stress concentrations around the holes. These sections were able to resist torques much higher than the torque at the onset of yield, but at the expense of severe plastic deformation around the hole.

The effect of the diameter of the hole was seen to be important. Small holes had little effect on the overall resistance but decreased the extent of plastic behaviour. Larger holes caused greater reductions in resistance and stiffness, and, in most cases, resulted in negative stiffness at high twist associated with collapse at the location of the opening.

A double hole was found to have a similar effect on stiffness and strength to that of a single hole of the same diameter. However, the negative stiffness at high rotation was greater for the specimens with two holes than it was for the corresponding single hole specimens (collapse was more rapid).

For the small-scale tests, the specimens without holes (Fig. 6a) were seen to behave as predicted by thick wall torsion theory (based on $0.2 \%$ proof stress). The initial elastic behaviour was followed by a transition from fully elastic to fully plastic behaviour. The plastic deformation (around 15 times the elastic rotation) showed marginally positive stiffness, which was the result of strain hardening. This was followed by a significant drop in torque, associated with large out-of-plane distortion of the walls adjacent to the end of one of the metal inserts.

However, the results of the full-scale torsion tests indicated that the unperforated sections were unable to achieve even their thick-wall elastic torsional capacities. The maximum torques were some $20 \%$ lower than the predicted plastic capacities $\left(T_{p l}\right)$ in the case of the grade S275 specimens, and some $12 \%$ lower in the case of the S355 specimens. Despite the poor agreement with the theory, the two tests of unperforated grade S355 RHS 200x100x8 gave similar results indicating good experimental repeatability (less than 1\% difference in capacity). The possible causes of the anomalous torsional behaviour of the full-scale unperforated specimens, which is also evident in the work of Marshall (1972), is discussed further elsewhere (Ridley-Ellis et al $2002 \& 2003)$.

\subsection{Measurements and FEA for the full-scale tests}

Figures 4 and 5 also show the torque-rotation relationships predicted by the FEA models. In general, the agreement between the relationships measured by the full-scale tests and those predicted by FEA was good. However, the measured torsional capacities of the sections without wall openings were substantially lower than those predicted by FEA.

The predictions of the FEA models of the unperforated specimens were virtually identical to those of thick wall theory. The long yield plateau of the hot-finished steel meant that the shear strains were insufficient for significantly strain-hardened stresses to develop and that the FEA predictions of torsional capacity were limited to the theoretical plastic torsional capacity. None of the laboratory specimens were susceptible to shear buckling of the tube wall prior to development of the plastic torsional capacity, although the FE model of the grade S355J2H RHS 200x100x8 exhibited slight buckling behaviour at high rotation.

This agreement between the FEA and the predictions of thick wall theory is in contrast to the actual experimental results. The unperforated RHS tested in the laboratory failed to achieve even their predicted elastic torsional capacities.

In all cases of the perforated sections, the FEA prediction of initial stiffness was within $15 \%$ of the measured value, and in 7 of the 9 cases it was within $6 \%$. In all but one case, the torque predicted at $\theta_{e l}$ was within $5 \%$ of the measured value, and for that one exception the difference was only $13 \%$.

The torque rotation relationships predicted by the FEA models were extremely close to the experimental measurements for the grade S355 specimens with the large holes. The comparison for the grade S275 specimens was not as good, with the FEA predicting, in general, a somewhat stiffer overall torsional response and a slightly higher torques for the larger values of twist. However, in all the cases with large holes, the FEA models were able to correctly predict the general shape of the curve, and good matches were obtained at the lower values of twist and with respect to stress patterns and deformations around the openings (Figs 8 and 9).

For the case of the RHS with two small holes, the FEA prediction and the experimental result were similar, with FEA predicting the correct elastic response, but a higher maximum torque and a reduced torsional ductility that was not observed in the tests. In the FEA model, the negative stiffness after achieving the maximum torque was due to buckling at the location of the opening, which did not occur in the laboratory.

\subsection{Measurements \& FEA for the small-scale tests}

Figure 6 also shows the torque-rotation relationships predicted by the FEA models of the small-scale tests. The agreement between the FEA predictions and the measured values is extremely good with a maximum difference in peak torque of only $3 \%$. 


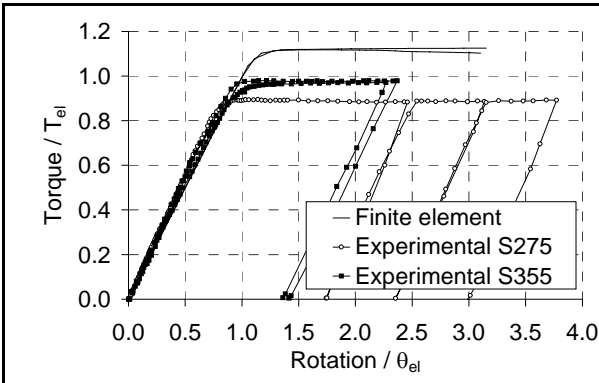

(a) No holes $\left(\mathrm{T}_{\mathrm{pl}}=1.12 \times \mathrm{T}_{\mathrm{el}}\right)$

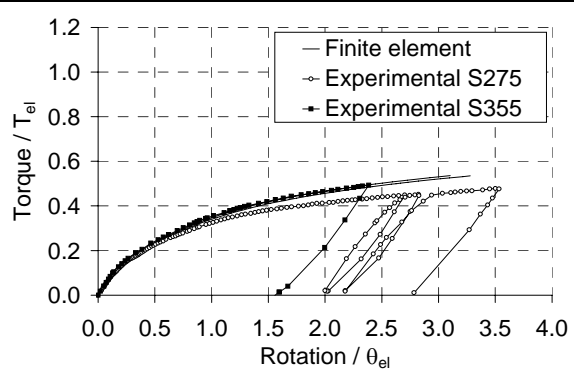

(b) One hole, Dia $165 \mathrm{~mm}$

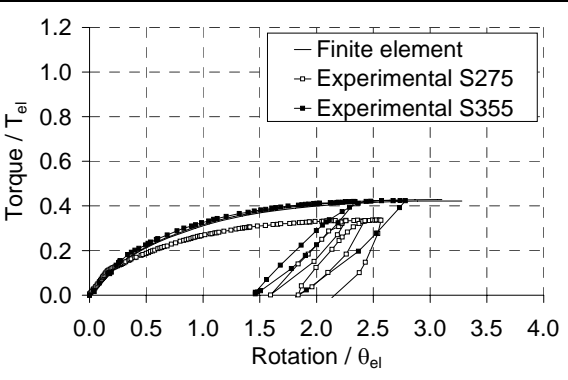

(c) Two holes, Dia $165 \mathrm{~mm}$

Figure 4. Torque-rotation relationships (experimental and finite element) for RHS 200x100x8.



(a) No holes $\left(\mathrm{T}_{\mathrm{pl}}=1.08 \times \mathrm{T}_{\mathrm{el}}\right)$



(b) One hole, Dia $99 \mathrm{~mm}$

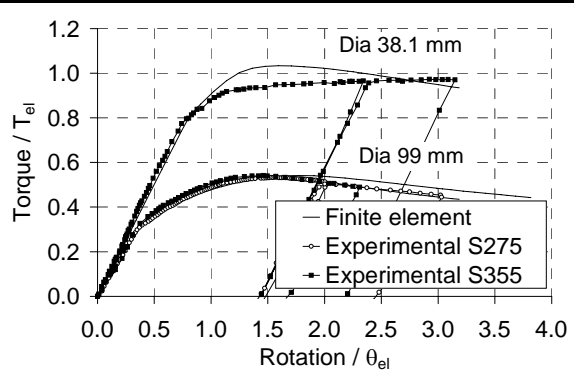

(c) Two holes, Dia $38.1 \& 99 \mathrm{~mm}$

Figure 5. Torque-rotation relationships (experimental and finite element) for RHS 150x150x6.3.

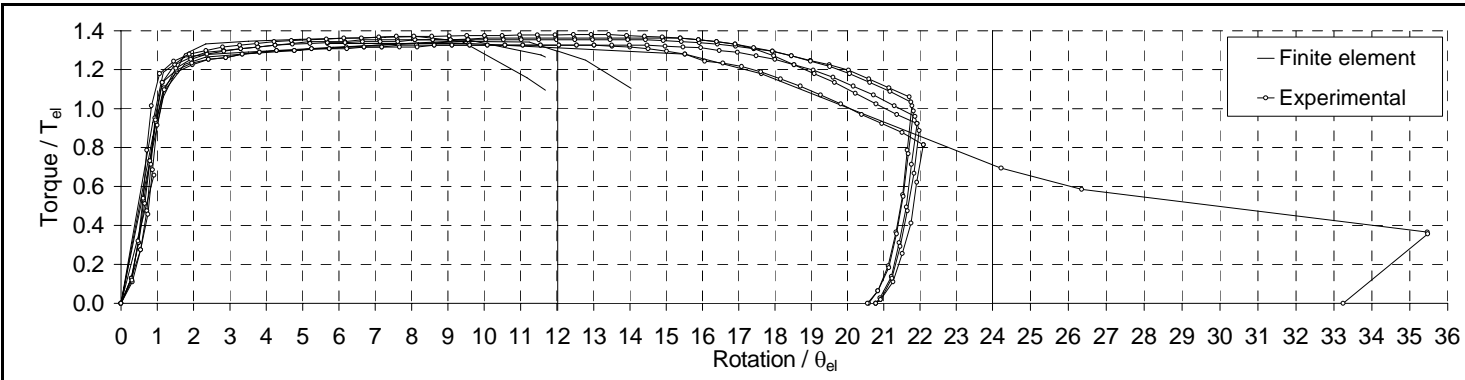

(a) No holes $\left(\mathrm{T}_{\mathrm{pl}}=1.08 \times \mathrm{T}_{\mathrm{el}}\right)$



(b) One hole, Dia 5 \& $20 \mathrm{~mm}$

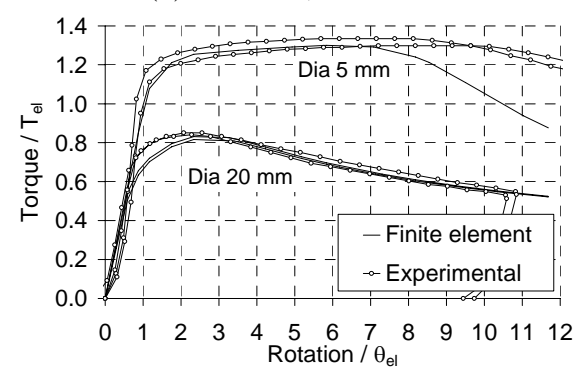

(e) Two holes, Dia 5 \& $20 \mathrm{~mm}$

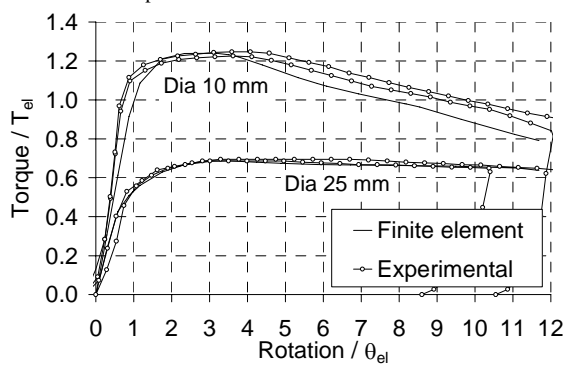

(c) One hole, Dia $10 \& 25 \mathrm{~mm}$

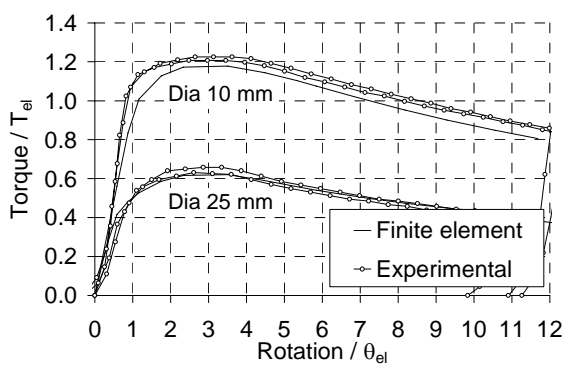

(f) Two holes, Dia $10 \& 25 \mathrm{~mm}$



(d) One hole, Dia 15 \& $30 \mathrm{~mm}$

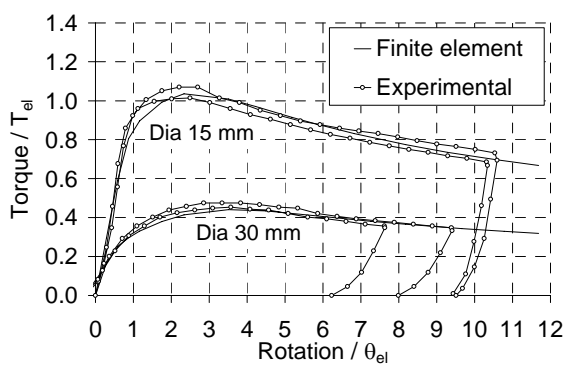

(g) Two holes, Dia 15 \& $30 \mathrm{~mm}$

Figure 6. Torque-rotation relationships (experimental and finite element) for small-scale torsion-tests. 




Figure 7. Examples of deformed perforated zones (smallscale).

The good agreement is true for the entire torquerotation relationship with FE correctly modelling the onset of non-linearity, the maximum torque and the plastic collapse. As was the case with the full-scale tests, good matches were obtained with respect to deformations around the openings (Figs 7 and 10) The largest difference occurred for the extent of torsional ductility (onset of plastic collapse) of the unperforated specimens (Fig. 6a), which was found to be particularly sensitive to the FEA mesh density.

The agreement in the torque at yield and maximum torque for the unperforated specimens and those with small holes is in contrast to the large differences observed at full-scale (Figs 4a and 5a).

The FEA predictions of initial elastic stiffness were generally slightly lower than the experimentally measured values. This is thought to be due to the low values of Young's modulus used in the FEA material models (Section 3.2). However, accurate experimental measurements of initial stiffness were difficult, due to limitations of the testing method.

\subsection{The influence of boundary conditions}

The boundary conditions used in the FEA models were found to be important for unsymmetrical cases. Figure 11 shows how, for a single hole diameter, three FEA models can give very different results depending on the boundary conditions at the ends of the specimen. When the boundary conditions are fixed in both transverse directions at both ends ('fixed'), the member is able to reach a higher value of peak torque than if the boundary conditions are fully pinned ('pinned'). Fixed boundary conditions resist the tendency of the unsymmetrical specimens to bend at high twist.

The influence of boundary conditions is more significant for the small-scale specimens than the fullscale torsion specimens because the values of twist are higher and the specimens are shorter in relation to the section depth. For the small-scale tests the actual support conditions were that the ends were pinned in one transverse direction and fixed in the other such that the pinned axes at each end were perpendicular to one another ('cross-pinned'). This condition was selected to prevent accidental transmission of bending to the laboratory specimen. The cross-pinned condition meant that the specimen reached a maximum torque between that of the pinned and fixed cases, and was able to maintain the torque with increasing twist.

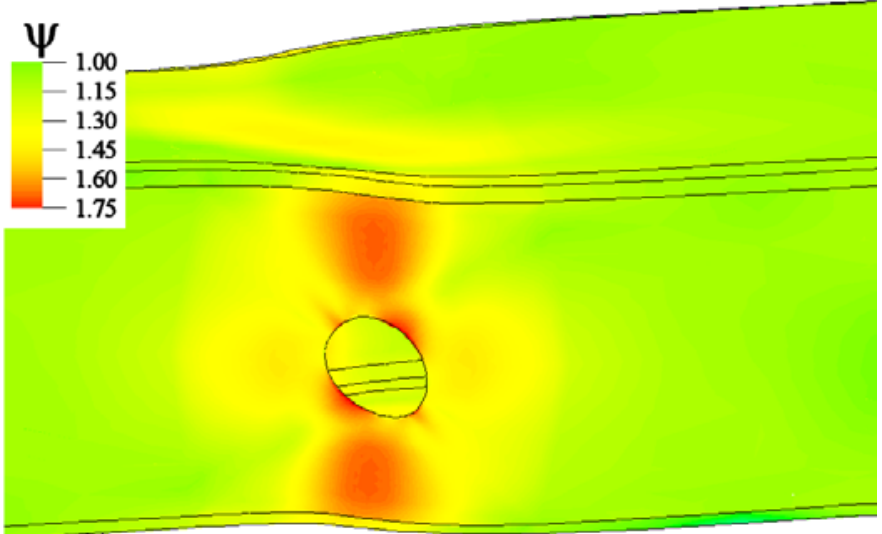

$\Psi=$ Max von Mises at hole / Max von Mises away from hole

Figure 8. FEA concentrations of von Mises stress for full-scale specimen with $38.1 \mathrm{~mm}$ hole (RHS 150x150x6.3 S355).

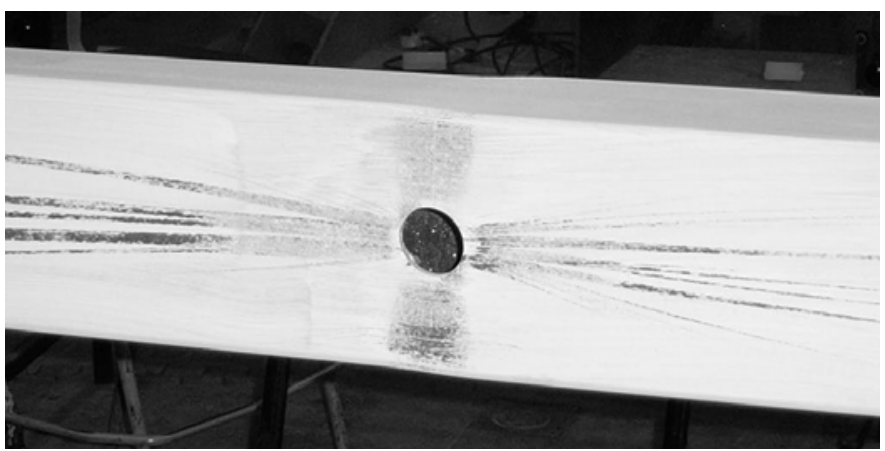

Figure 9. Lüders lines on full-scale test with $38.1 \mathrm{~mm}$ hole.



Figure 10. Examples of FEA deformed shapes (small-scale).




Figure 11. The influence of boundary conditions (FEA models of small-scale test with one $30 \mathrm{~mm}$ hole).

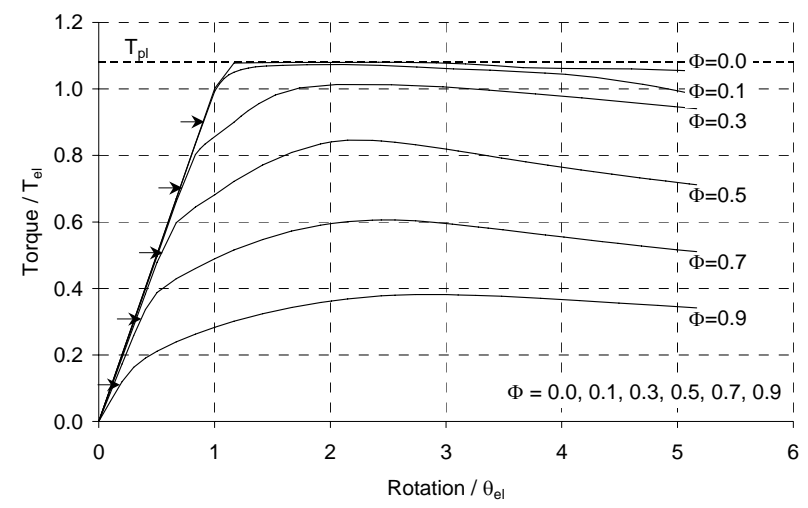

Figure 12. Torque-rotation relationships for RHS 250x250x10 with two holes of various diameters (FEA).

\subsection{Observations from the parametric FEA study}

The FEA parametric study was conducted to provide further information about stress concentration, and the importance of relative hole diameter and tube aspect ratio. A non-dimensional quantity $(\Phi)$ was used to indicate the relative size of the perforation. This is the ratio of the hole diameter to the depth of the flat part of the perforated wall (i.e. not including corner radii). Values of $\Phi$ for the experimental specimens are given in Tables 1 and 2.

The results of the parametric study were consistent with the results of the experimental work in showing that holes cause a reduction in both torsional capacity and stiffness, and that these reductions are greatest for holes that remove a large portion of the depth of the tube wall.

The presence of the opening was observed to cause an increased von Mises stress at the edge of the hole, due to the reduced area of cross-section and the stress concentrating effect of the change in crosssection. For small holes, the small amount of yielding that occurs around the opening was seen not to have a significant effect on the initial torque-rotation behaviour of the member. The raised stress that occurs around larger holes was seen to have a more significant effect resulting in a more gradual transition from a linear response to zero stiffness. Perforations were also seen to reduce the torsional ductility of a member, although RHS with very large holes were seen to be able to maintain their peak torque for a slightly larger twist than equivalent section with holes of intermediate size (e.g. Fig. 12).

The results of the parametric FEA study indicated that the point at which the torque-rotation relationship ceases to be linear is only slightly dependent on the aspect ratio of the RHS. The relative reduction in the torque at onset of this non-linearity was found to be approximately equal to the fraction of the wall depth removed by the opening $(\Phi)$. For the sections chosen for the parametric study, this method of approximating the reduced elastic response (indicated by arrow symbols on Figure 12) was found to be conservative.

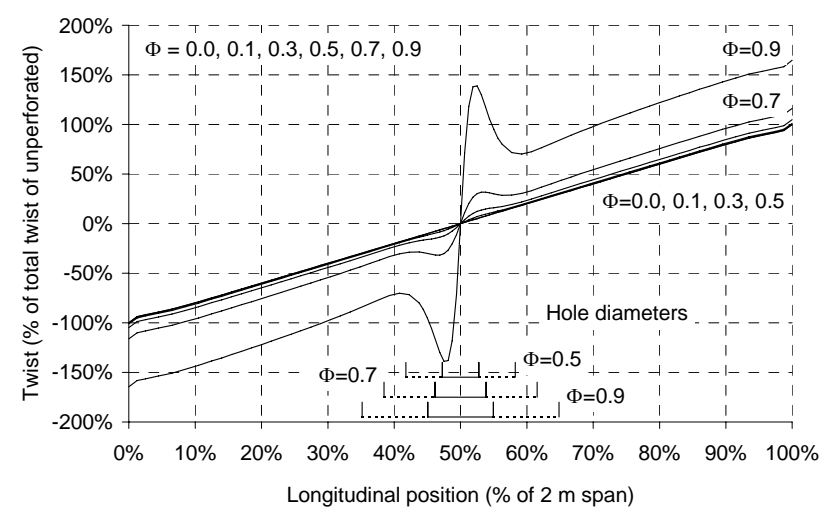

Figure 13. Twist across the perforated zone for RHS 250x250x10 with two holes of various diameters (FEA).

The reduction in the torsional stiffness of a perforated RHS was seen to be due to large deformations occurring within a zone that is equal in length to three times the diameter of the hole (e.g. Fig. 13). In the elastic range, it was found that the twist across this perforated zone could be estimated by applying a reduced stiffness over a length equal to the depth of the perforated wall. This reduced stiffness being the product of the theoretical stiffness of the unperforated RHS and the factor $\rho$ given in equation 1 . This method was conservative for all the sections chosen for the parametric study (Section 3.3).

$\rho=1 /(1-\Phi)$

An exploratory FEA study of 73 further models was also conducted to quantitatively study the stress concentrating effect of the perforations. This study, which looked at a wide range of perforated RHS under small elastic torques, concluded that the stress concentrating effect of a perforation is dependent on the wall slenderness and the hole size, but not the aspect ratio of the tube.

An unperforated RHS in pure elastic torsion has a distribution of elastic shear stress that varies through the thickness and is greater (typically 20 to 30\%) on the external surface. A small circular opening on the neutral axis disturbs this system of stress and produces a stress pattern similar to that around a hole in an infinite plate in pure shear. For small holes $(\Phi<$ 0.3 ) the hole was found to cause a small area of raised von Mises stress that was around twice the maximum von Mises stress away from the perforated region. For very large holes in slender walls, this factor of concentration ( $\Psi$ ) was much higher. Table 3 shows approximate values of $\Psi$ for a range of hole size ratios and perforated wall slendernesses. The stress concentration in an RHS with one hole was found to be nearly identical to that in an RHS with two holes, although the maximum stress was 
seen to be slightly higher (around 10\%) for sections with two holes of large diameter.

Table 3. Approximate factors for the concentration of von Mises stress around a perforation ( $\Psi)$.

\begin{tabular}{llllllllll}
\hline Hole size & \multicolumn{10}{c}{ Slenderness } & of perforated wall \\
ratio $(\Phi)$ & 12 & 14 & 16 & 18 & 20 & 22 & 24 & 26 & 28 \\
\hline 0.1 & 2 & 2 & 2 & 2 & 2 & 2 & 2 & 2 & 2 \\
0.2 & 2 & 2 & 2 & 2 & 2 & 2 & 2 & 2 & 2 \\
0.3 & 2 & 2 & 2 & 2 & 2 & 2 & 2 & 3 & 3 \\
0.4 & 2 & 2 & 3 & 3 & 3 & 3 & 3 & 3 & 3 \\
0.5 & 3 & 3 & 3 & 3 & 3 & 3 & 3 & 3 & 3 \\
0.6 & 3 & 3 & 3 & 3 & 3 & 4 & 4 & 4 & 4 \\
0.7 & 3 & 4 & 4 & 4 & 4 & 4 & 5 & 5 & 5 \\
0.8 & 4 & 5 & 5 & 5 & 6 & 6 & 7 & 7 & 7 \\
0.9 & 7 & 8 & 9 & 10 & 11 & 11 & 12 & 13 & 14 \\
\hline
\end{tabular}

\section{CONCLUDING REMARKS}

A series of 14 full-scale torsion tests were conducted on two sizes and grades of hot-finished RHS to assess the effect of wall openings upon structural performance in torsion. A further 30 small-scale torsion tests were conducted on one size of cold-formed steel tube to provide additional information about the influence of relative hole diameter. These laboratory tests were supplemented by FEA modelling of the tests conducted, and a parametric FEA study to widen the scope of the investigation.

The experimental results were compared with FEA predictions and good agreement was observed in terms of displaced shapes, elastic stiffness and strains in elastic range. Experimental torque-rotation relationships matched closely those predicted by the FEA models in the perforated cases, but were significantly different for the unperforated full-scale specimens.

The experimental results showed that large holes cause a significant reduction in both torsional capacity (up to 60\%) and stiffness (up to 40\%). The reduction in stiffness was due to the perforated zone being much more flexible than the unperforated beam, and severe deformations in this region were observed. The FEA parametric study indicated that the reduction in stiffness across the perforated zone was approximately proportional to the fraction of the wall depth removed by the opening.

The holes with the greatest diameter caused the biggest reduction in resistance and stiffness and resulted in yielding of material occurring at lower twist. Small holes caused only a small reduction in the torsional resistance and stiffness, but reduced the torsional ductility of the member. The presence of the opening was observed to cause an increased von Mises stress in the walls at the edge of the opening, due to the reduced area of cross-section and the stress concentrating effect of the change in crosssection.

Although, perforated RHS were seen to be able to continue to resist increasing torque following yield- ing of large areas of material around the holes, this was observed to occur at the cost of large deformations across the perforated zone and a consequent reduction in stiffness. The results of the FEA parametric study indicated that the reduction in the torque at onset of non-linearity in the torque-rotation relationship is approximately proportional to the fraction of the wall depth removed by the opening.

The FEA models also showed that, for unsymmetrical cases, the boundary conditions at the ends have a large influence upon the torsional behaviour of short members.

With the exception of the FEA models used to specifically study stress concentrations, the RHS chosen in the experimental and FEA investigations described in this paper were of sufficient wall thickness that unperforated sections were able to achieve their fully plastic moment without instability.

\section{REFERENCES}

EN 10210-2:1997. 1997. Hot finished structural hollow sections of non-alloy and fine grain structural steels: Tolerances, dimensions and sectional properties. European Committee for Standardization.

HKS. 2000. ABAQUS/Standard version 5.8-1 (computer program). Hibbitt, Karlsson and Sorensen Inc, Pawtucket, Rhode Island, USA.

Marshall, J. 1972. Aspects of torsion of structural rectangular hollow sections, Ph.D. thesis. University of Strathclyde.

Mullett, D.L. 1997. Design of RHS Slimflor edge beams. Steel Construction Institute, Ref. SCI-P-169.

Ridley-Ellis D. J., Owen J. S. \& Davies G. 2003. Torsional behaviour of Rectangular Hollow Sections. Journal of Constructional Steel Research. (in press).

Ridley-Ellis D. J., Owen J. S. \& Davies G. 2002. Theoretical and measured torsional behaviour of Rectangular Hollow Sections. In J.S. Chung, M. Sayed, M. Kashiwagi, T. Setoguchi, S.W. Hong (eds), Proc. of the 12th Intern. Offshore and Polar Engineering Conf. (ISOPE), Kyushu, May 2002. 4: 41-47. International Society of Offshore and Polar Engineers. ISBN1-880653-58-3, ISSN1098-6189.

Ridley-Ellis D. J. 2000. Rectangular Hollow Sections with circular web openings. PhD Thesis. The University of Nottingham. British Library Shelfmark DXN042674.

Ridley-Ellis D. J., Davies G. \& Owen J. S. 1998. Fundamental behaviour of rectangular hollow sections with web openings. In Y.S. Choo and G.J. van der Vegte (eds), Proc. of the 8th Intern. Symp. on Tubular Structures (ISTS), Singapore, August 1998. Tubular Structures VIII: 167-175. Rotterdam: Balkema. ISBN 9058090019.

\section{ACKNOWLEDGEMENT}

The authors are grateful to Corus Tubes and Nottingham University for their valuable support. 